\title{
CURSO DE INFORMÁTICA RELACIONADO AOS ASPECTOS COGNITIVOS DA PESSOA IDOSA: UMA REVISÃO SISTEMÁTICA
}

\section{Computer Course Related to Cognitive Aspects of Elderly People: a Systematic Review}

\author{
Luana Suemi Fujita ${ }^{1}$ \\ Gerson Flores Gomes ${ }^{2}$ \\ Jaqueline dos Santos Valente Barros ${ }^{3}$ \\ Valdomiro de Oliveira ${ }^{4}$ \\ Gislaine Cristina Vagetti ${ }^{5}$
}

\begin{abstract}
Resumo: Os cursos de informática para pessoas idosas podem propiciar um desempenho satisfatório de suas habilidades cognitivas, postergando os problemas decorrentes dos transtornos neurocognitivos que surgem nesta fase da vida. O objetivo do presente estudo foi revisar sistematicamente evidências dos aspectos cognitivos da pessoa idosa relacionado ao curso de Informática. A revisão sistemática da literatura seguiu os princípios da declaração PRISMA. As bases de dados utilizadas nesta revisão foram: BVS, ERIC, Scielo, Science Direct, PsynINFO, Web of Science e Scopus, com os seguintes descritores: idosos, tecnologia, cognição, nas línguas portuguesa, inglesa e espanhola. Foram selecionados artigos de observação relacionados a cognição de pessoas com 60 anos ou mais e o curso de informática publicados no período de janeiro de 2009 a dezembro de 2019. A qualidade dos artigos foi verificada por dois revisores independentes por meio do The Reporting of Observational Studies in Epidemiology - STROBE. Apenas nove estudos preencheram os critérios de inclusão, abordando a cognição relacionada ao curso de informática. Considerando a revisão dos estudos, não foi possível afirmar que a inclusão digital, a partir das diversas tecnologias disponíveis, causa efeitos positivos para a cognição dos idosos. A quantidade de estudos encontrados com o uso dos critérios de inclusão/exclusão resultantes desta revisão, apurada nas bases de dados pesquisadas, foi pequena considerando-se a importância do assunto.
\end{abstract}

Palavras-chave: Revisão Sistemática. Idosos. Curso de Informática.

\footnotetext{
${ }^{1}$ Mestre em Educação, na linha de Cognição, Aprendizagem e Desenvolvimento Humano pela Universidade Federal do Paraná. Licenciada em Pedagogia pela Universidade Estadual de Ponta Grossa. https://orcid.org/0000-0001-8373-631X. E-mail: luana_1982@hotmail.com

${ }^{2}$ Doutorando em Educação pela Universidade Federal do Paraná. Mestre em Educação na linha de Cognição, Aprendizagem e Desenvolvimento Humano pela Universidade Federal do Paraná. https://orcid.org/0000-00033686-7207. E-mail: ggomes.ufpr@gmail.com

3 Doutora em Educação pela Universidade Federal do Paraná. Docente na Universidade Federal do Acre. https://orcid.org/0000-0003-3686-7207. E-mail: jaqueline.ufac@hotmail.com

${ }^{4}$ Doutor em Educação Física, Programa de Pós-Graduação em Educação, Universidade Federal do Paraná. https://orcid.org/0000-0002-8709-8471E-mail: oliveirav457@gmail.com

${ }^{5}$ Doutora em Educação Física, Universidade Estadual do Paraná.https://orcid.org/0000-0003-0704-1297. E-mail: gislainevagetti@hotmail.com
} 
Abstract: Computer courses for elderly people can provide a satisfactory performance of their cognitive skills, postponing the problems resulting from neurocognitive disorders that arise at this stage of life. The aim of the present study was to systematically review evidence of the elderly person's cognitive aspects related to the Informatics course. The systematic review of the literature followed the principles of the PRISMA statement. The databases used in this review were: VHL, ERIC, Scielo, Science Direct, PsynINFO, Web of Science and Scopus, with the following keywords: elderly, technology, cognition, in Portuguese, English and Spanish. Observation articles related to the cognition of people aged 60 or over and the computer course published between January 2009 and December 2019 were selected. The quality of the articles was verified by two independent reviewers through the Strengthening the Reporting of Observational Studies in Epidemiology - STROBE. Only nine studies filled in the inclusion criteria, addressing cognition related to the computer course. Considering the review of the studies, it was not possible to state that digital inclusion, based on the various available technologies, causes positive effects on the cognition of the elderly. The number of studies found using the inclusion / exclusion criteria resulting from this review, found in the researched databases, was small considering the importance of the subject.

Keywords: Systematic review. Seniors. Computer course.

\section{Introdução}

Em geral, os estudos sobre o desenvolvimento da humanidade privilegiam principalmente as primeiras fases da vida humana, como a fase infantil, a adolescência e a juventude, para as quais existe uma extensa literatura em diversas áreas do conhecimento. Nos últimos anos, pesquisadores têm se debruçado sobre a temática da vida adulta e da fase idosa (VAGETTI, et al., 2013), motivados pelos resultados de pesquisas demográficas que ressaltam mudanças no crescimento populacional, apresentando projeções para o crescimento do envelhecimento etário da população mundial. Com base neste contexto e lacuna, o objetivo deste estudo foi elaborar uma revisão sistematizada sobre a relação do curso de informática com os domínios da cognição em idosos.

O envelhecimento humano é uma etapa natural da vida e uma grande parcela da população desta faixa etária padece com declínio de suas capacidades cognitivas, prejuízos que afetam a qualidade de vida dos idosos, limitando sua autonomia, capacidade de tomada de decisão e seu bem-estar (ARGIMON; STEIN, 2005). Ao longo da vida dos seres humanos, o desenvolvimento e a manutenção das funções cognitivas permanece ativo desde que o sujeito esteja em plena atividade destas habilidades ou experimentando novas atividades (WILD et al., 2012). A informática, com o passar dos anos, tem se comprovado uma ferramenta com diversas possibilidades de atuação. Tem também se mostrado auxiliadora na medida em que ajuda a desenvolver novos modelos de organização da vida, com a otimização do tempo para realização de trabalhos e tarefas (KACHAR, 2003).

A tecnologia transforma os modos de comunicação e de interação social bem como o cotidiano das pessoas. Quando um indivíduo se conecta ao mundo tecnológico e virtual, ele vivencia um processo de construção e desconstrução de identidade diante da multiplicidade dos aspectos sociais e culturais (TARALLO; SÉ, 2016). As autoras afirmam que é necessário dominar os diversos recursos tecnológicos existentes que permeiam a vida dos indivíduos nas mais variadas faixas etárias. Nessa perspectiva, aqueles que não utilizam ou não têm acesso a essas tecnologias, por vezes, acabam sendo excluídos desses processos de mudanças.

O estudo de Pereira e Neves (2011) sugere que há uma grande aceitação das novas tecnologias pela população idosa e, além disso, que essa população percebe que o aprendizado 
destas novas tecnologias pode trazer potenciais benefícios, em especial para a manutenção das conexões sociais, das funções cognitivas e da qualidade de vida em geral. Entretanto, Scoralick-Lempke, Barbosa e Mota (2011), admitem que alguns fatores desencorajam os idosos no uso das tecnologias de informação e comunicação (TIC), como por exemplo, a autopercepção dos declínios naturais dos sentidos e das habilidades motoras, as mudanças na memória de trabalho e a redução do convívio social.

Foram encontradas pesquisas relacionadas à inclusão digital de idosos, com o desenvolvimento de cursos e oficinas de informática. Estes lócus de pesquisa podem ser definidos como espaços pedagógico-teórico-práticos, criados para a vivência, reflexão e construção do conhecimento (SILVEIRA, 2015). No rol de estudos da inclusão digital com pessoas idosas, é ressaltada a importância desse sujeito desenvolver estratégias para que tenha domínio no uso dessas ferramentas, o que lhe possibilitará acesso às informações e à execução de diversas atividades de natureza pessoal e profissional no cotidiano (SANTOS; ALMÊDA, 2017).

Diversas pesquisas (KACHAR, 2010; LEMOS et al., 2011; OLIVEIRA; SCORTEGAGNA; OLIVEIRA, 2015, FLORES-GOMES et al., 2020) revelam o perfil dos novos idosos que, mesmo estigmatizados e com todos os limites (visual, motor, auditivo e cognitivo) impostos pela idade, estão reinventando essa fase, buscando novos conhecimentos e novas aprendizagens por meio de vários cursos e, principalmente, cursos de informática. Dentre as múltiplas ferramentas que podem promover o envelhecimento saudável, o uso do computador e de outras tecnologias de informação e comunicação têm se mostrado promissoras (SCORALICK- LEMPKE; BARBOSA; MOTA, 2012).

\section{Inclusão digital na Terceira Idade}

Esta 'revolução da informação' envolve recursos eletrônicos e outras questões digitais, permitindo que o indivíduo possa estar mais integrado a um novo modelo de comunidade e de sociedade,mais dinâmicas e com relações puramente virtuais, colocando-o em contato com pessoas que estão distantes fisicamente, colaborando com a troca rápida de informações e com uma aprendizagem continuada. Concernente aos idosos, além dos aspectos desenvolvimentistas citados adquiridos por meio da inclusão digital, promove-se também uma nova cidadania digital, permitindo a integração, a efetividade e a interação no mundo.

Com a inserção das Tecnologias Digitais da Informação e Comunicação (TDIC) na sociedade contemporânea, faz-se necessário o desenvolvimento de novas habilidades pessoais para o uso destas tecnologias, ganhando novos sentidos. Os indivíduos, uma vez conectados, podem fazer contato com outras realidades e produzir conhecimentos relevantes para sua subsistência social. Nesta direção, o processo de envelhecimento humano foi sendo ressignificado, pela mudança de visão de si mesmo, enquanto indivíduo deste mundo. Apesar das perdas físicas e emocionais dos idosos, concordamos com Kachar (2003), em sua afirmação de que, nessa etapa da vida, é possível descobrir novos sonhos, projetos e desejos. Neste sentido, as questões da velhice exigem um novo olhar pelo idoso e pela sociedade.

Uma busca sistematizada foi feita como parte do referencial teórico da dissertação de Fujita (2020), sendo encontrado apenas nove estudos, no período de 2009 a 2019, com referência específica para este tema. Os achados destes estudos são discordantes, muitos apresentando efeitos positivos e outros negativos da inclusão digital dos idosos.

Cada indivíduo, diante das novas tecnologias, possui necessidades específicas, expectativas próprias, realidades diferenciadas, habilidades e conhecimentos já adquiridos.Em geral, a elaboração de novas tecnologias tem seu desfecho na sociedade, favorecendo o 
desenvolvimento humano (FLORES-GOMES et al., 2020). O aumento da velocidade de comunicação, do processamento e armazenamento destas informações, a criação de novos produtos inteligentes e seu impacto nas ciências é resultado destes avanços tecnológicos (ALVARENGA; YASSUDA; CACHIONI, 2019).

Os pesquisadores Cardoso et al.(2014) afirmam que a internet tem características positivas, permitindo a comunicação a distância e diminuindo o sentimento de solidão, o que pode influenciar no desenvolvimento de mudanças cognitivas e sociais nessa fase idosa da vida. $\mathrm{O}$ processo de aprendizagem está ligado às funções cognitivas, em especial a memória, já que este processo compreende mudanças de comportamento que se tornam permanentes. A memória abarca o processo de aprendizagem de novas informações, o armazenamento e a disponibilidade de acesso a esses conhecimentos (ALVARENGA; YASSUDA; CACHIONI, 2019).

Corroborando, os autores Flores-Gomes et al. (2020), afirmam que construir estratégias de abordagens educacionais, visando preparar pessoas idosas no domínio operacional dos recursos tecnológicos, gerar a alfabetização de novas linguagens, que hoje já se fazem presentes em todos os setores da sociedade e promover a inclusão do idoso na sociedade digital, são questões importantes que podem gerar intervenções e pesquisas científicas. Enquanto a alfabetização digital se refere ao processo inicial de aprender a utilizar o computador, o domínio da ferramenta, o letramento digital envolve competências como compreender, assimilar, reelaborar, adaptar e construir o conhecimento, a partir de práticas de leituras, releituras de informações, trocas interacionais virtuais e a escrita destas comunicações, a fim de utilizar as Tecnologias da Informação e Comunicação para benefício na vida pessoal e coletiva destes idosos.

\section{Cognição, Educação e Envelhecimento}

Estudos sugerem que a cognição é formada por quatro funções básicas: velocidade de processamento, memória de trabalho, inibição e função sensorial e que ainda não há linearidade para o estabelecimento de um constructo para o declínio cognitivo relacionado à idade, tendo em vista que o envelhecimento é diferencial (NUNES, 2009). Define-se cognição, como um conjunto de processos mentais usados no pensamento, reconhecimento e compreensão, para o julgamento por meio do raciocínio e do aprendizado (FONSECA, 2014). Em seu estudo, os pesquisadores Ordonez, Yassuda e Cachioni (2011), observaram ganhos cognitivos em idosos que participaram de um processo de inclusão digital denominado Idosos On-Line. Os pesquisadores verificaram que o grupo experimental, ao ser comparado com um grupo controle, apresentou no pós-teste um aumento significativo em linguagem e na memória.

Sabe-se que algumas funções biológicas tendem a declinar com a idade e a preservação do desempenho cognitivo frente ao processo de envelhecimento pode ser um determinante crítico da qualidade de vida na velhice e de uma maior longevidade (KRAMPE et al., 2011). Com o processo de envelhecimento, alguns subsistemas da memória sofrem alterações significativas. Segundo o estudo de Parente, Oliveira e Jaeger (2006), estes encontraram que os déficits de memória em indivíduos estão associados com dificuldades na codificação e na recuperação das informações. Na busca de soluções para manutenção destas capacidades relativas à cognição, pesquisadores têm se debruçado sobre o uso de novas formas para aprendizagem das tecnologias da informação e comunicação, as TIC, e sobre o uso de computadores e outros equipamentos que possam gerar a estimulação cognitiva e melhorias nos domínios motores (ORDONEZ; YASSUDA; CACHIONI, 2011). 
O processo de envelhecimento populacional, as mudanças da longevidade humana, a explosão demográfica e a abertura do horizonte geográfico levantam importantes reflexões em todos os âmbitos da sociedade e precisam ser compreendidos em suas diversas peculiaridades. Essas reflexões conduzem os idosos a uma situação dinâmica de aprendizagem, pois a vida em si, independente da etapa da vida que se esteja, apresenta condições para novos aprendizados. Sendo assim, saber envelhecer de forma bem-sucedida é uma nova exigência da sociedade e do mundo.

\section{Método}

Trata-se de um estudo de revisão sistemática da literatura. Para elaborar a revisão, foram percorridas as seguintes etapas: 1) Definição da pergunta; 2) Definição da estratégia de busca; 3) Definição dos critérios de inclusão e exclusão dos artigos; 4) Busca dos estudos; 5) Seleção dos estudos; 6) Avaliação crítica dos estudos; 7) Apresentação dos resultados (SAMPAIO; MANCINI, 2007). A revisão sistemática sobre evidências dos aspectos cognitivos da pessoa idosa relacionados ao curso de Informática, seguiu os princípios da declaração PRISMA (Preferred Reporting Items for Systematic Reviews) de (GALVÃO et al., 2015). A busca compreendeu o período de janeiro de 2009 a dezembro de 2019 e alcançou sete bases de dados eletrônicas: Biblioteca Virtual da Saúde (BVS), Education Resources Information Center (ERIC), Scientific Eletronic Library Online (Scielo), Science Direct, PsycINFO, Web of Science e Scopus. Utilizaram-se os motores de busca das bases de dados, empregando-se os operadores booleanos AND e $O R$ com os descritores e sinônimos combinados: aged, aging, elderly, older adults, elder, cognition, cognitive skills, cognitive functions, technology, informatics, digital inclusion, informatics knowledge, social media e information technology.

A Figura 1 apresenta um fluxograma do processo de seleção de artigos, de forma visual, para que sejam melhor compreendidas as fases e etapas do método. O método utilizado possui três fases de trabalho. A fase "identificação", faz uso dos motores de busca das bases de dados, utilizando-se dos operadores booleanos AND e $O R$. Para a investigação foram utilizados os descritores encontrados nos Descritores em Ciências da Saúde (DeCS), Medical Subject Headings (MeSH) e Thesaurus. 


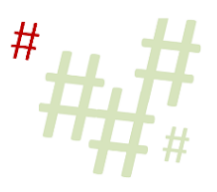

Figura 1 - Fluxograma da seleção de artigos

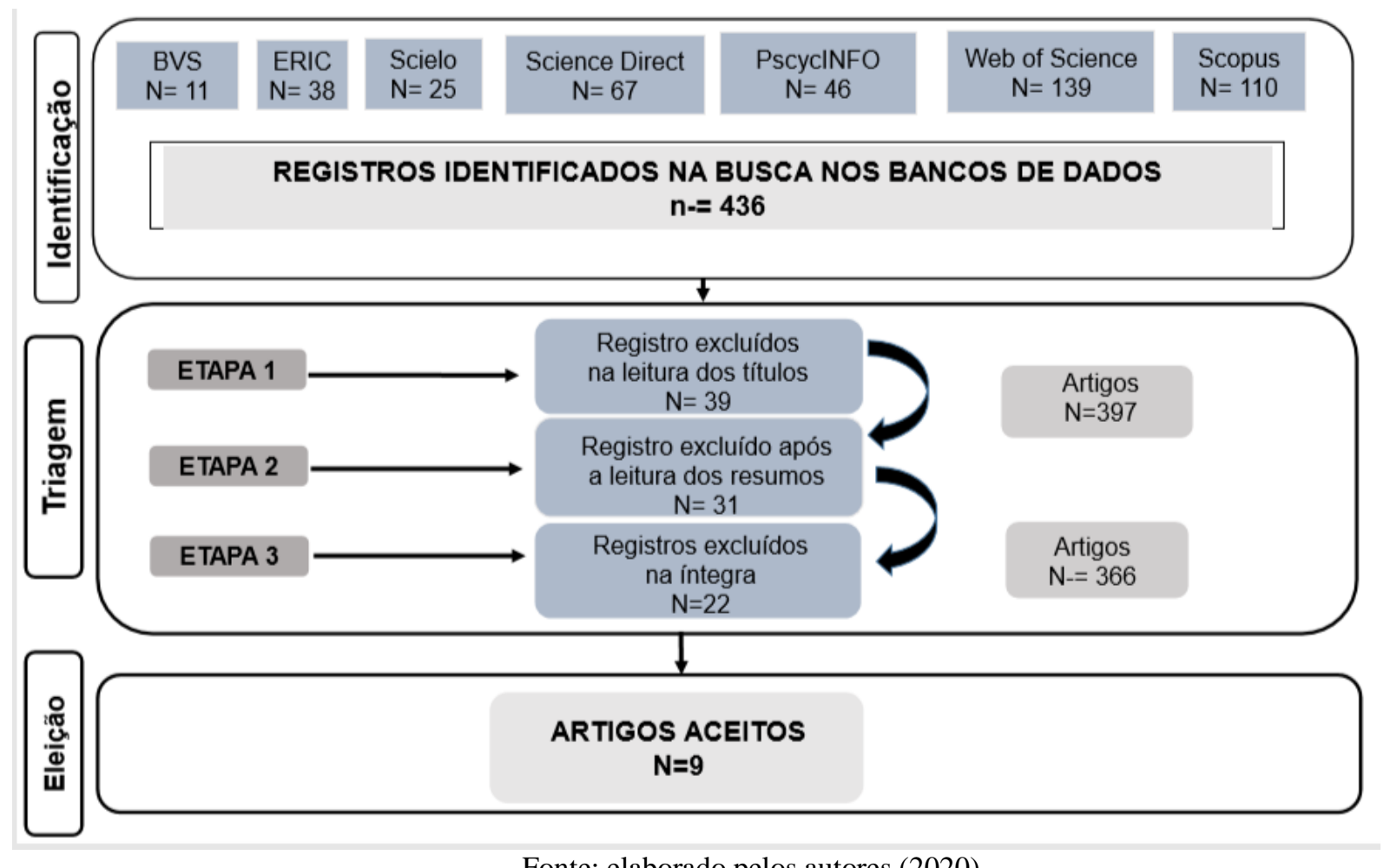

Fonte: elaborado pelos autores (2020)

$\mathrm{Na}$ fase "triagem", existem três etapas avaliativas que permitem a seleção de artigos. Na primeira etapa, são excluídos os estudos que não contemplam os critérios de inclusão e exclusão, especificamente pelos dados do campo 'título' do estudo. Na segunda etapa, são excluídos os artigos seguindo também os critérios de inclusão e exclusão, considerando a leitura dos resumos.

$\mathrm{Na}$ terceira etapa, é feita uma leitura integral dos estudos e revisão dos artigos selecionados por dois juízes, sendo feita uma qualificação dos estudos utilizando uma ferramenta de análise que, para este estudo, foi utilizado a lista de verificação de Downs \& Black (DOWNS; BLACK, 1998). Esse instrumento, avalia estudos de intervenção, possui alto grau de reprodutibilidade e consistência interna, além de contemplar grupos de comparação. O instrumento é composto por 27 questões, cada qual valendo no máximo um ponto, perfazendo o total de 27 pontos válidos. Cada revisor avalia todos os artigos e os pontua segundo a análise de cada questão e totaliza a nota para a qualidade do artigo, sendo estes escores informados na primeira coluna do Quadro 1, com a abreviação 'DB'.

$\mathrm{O}$ ponto de corte para eleição dos estudos foi validado para uma pontuação $\geq 18$ pontos e, caso haja alguma divergência entre as avaliações dos estudos, os revisores deveriam reavaliar os artigos e chegar a um consenso. Também nesta etapa foram excluídos todos os artigos que apresentaram duplicidade em bases de dados diferentes, sendo eleitos para inclusão os artigos que apresentaram registros únicos e exclusivos.

$\mathrm{Na}$ fase 'eleição', os artigos são sumarizados em um quadro, para uma melhor compreensão dos achados, considerando os autores, o ano de publicação, o local do estudo, os objetivos, o protocolo de intervenção aplicado, os instrumentos e os principais achados dos pesquisadores, conforme apresentado no Quadro 1.

Os critérios de inclusão de estudos para essa revisão foram: artigos que tenham pessoas idosas, participantes de programas de inclusão digital, oficinas de informática e 
Revista de Educação, Ciência e Tecnologia

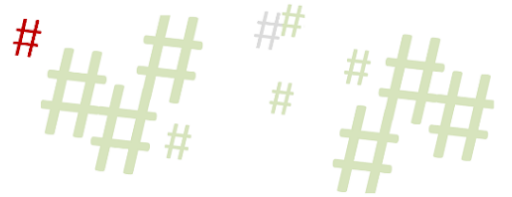

cursos de informática e estudos longitudinais de intervenção e observacionais com pesquisas realizadas no período de 2009 a 2019. Os critérios de exclusão foram estudos com idosos doentes ou para tratamento médico e estudos direcionados para profissionais de saúde - por exemplo, enfermeiros, cuidadores de idosos ou médico.

Quadro 1- Artigos encontrados nas bases BVS, ERIC, Scielo, ScienceDirect, PsycINFO, Web of Science e Scopus

\begin{tabular}{|c|c|c|c|c|c|c|}
\hline $\begin{array}{c}\text { Autor/ } \\
\text { Ano }\end{array}$ & País & Objetivo & Amostra & Intervenção & Instrumentos & $\begin{array}{c}\text { Principais } \\
\text { Achados } \\
\end{array}$ \\
\hline $\begin{array}{l}\text { Ordonez, } \\
\text { Yassuda \& } \\
\text { Cachioni } \\
\text { (2010) } \\
\text { DB: } 24\end{array}$ & $\begin{array}{l}\text { Brasil/ } \\
\text { São } \\
\text { Paulo }\end{array}$ & $\begin{array}{l}\text { Investigar os efeitos de } \\
\text { um programa de } \\
\text { inclusão digital no } \\
\text { desempenho cognitivo } \\
\text { de indivíduos mais } \\
\text { velhos que participaram } \\
\text { de um workshop de } \\
\text { aprendizado r de } \\
\text { computador chamado } \\
\text { "Idosos On-Line" } \\
\text { Conectados. }\end{array}$ & 42 idosos & $\begin{array}{l}\text { Os participantes } \\
\text { foram separados } \\
\text { em dois grupos - } \\
\text { um grupo } \\
\text { experimento e um } \\
\text { controle. r O } \\
\text { workshop incluiu } \\
\text { quinze lições de } \\
120 \text { minutos. }\end{array}$ & $\begin{array}{l}\text { 1.Quest.Soc.2.A } \\
\text { CE-R. }\end{array}$ & $\begin{array}{l}\text { Melhora } \\
\text { cognitiva } \\
\text { significativa } \\
\text { quanto } \\
\text { memória, à } \\
\begin{array}{l}\text { linguagem } \\
\text { habilidades } \\
\text { visuoespaciais. }\end{array}\end{array}$ \\
\hline $\begin{array}{l}\text { Pereira \& } \\
\text { Neves } \\
(2011) \\
\text { DB: } 18\end{array}$ & $\begin{array}{l}\text { Brasil/ } \\
\text { São } \\
\text { Paulo }\end{array}$ & $\begin{array}{l}\text { Aferir se o uso da } \\
\text { internet pode contribuir } \\
\text { para a infoinclusão } \\
\text { sênior e para a melhoria } \\
\text { da qualidade de vida, } \\
\text { expondo um processo } \\
\text { de alfabetização digital } \\
\text { para idosos. }\end{array}$ & 14 idosos & $\begin{array}{l}\text { Sessões } \\
\text { alfabetização } \\
\text { digital }\end{array}$ & $\begin{array}{l}\text { 1.Quest.Soc. } \\
\text { 2.Quest. } \\
\text { Aprendizagens } \\
\text { realizadas e } \\
\text { diminuição da } \\
\text { solidão e para o } \\
\text { aumento da QV. }\end{array}$ & $\begin{array}{lr}\text { Diminuição } & \text { da } \\
\text { solidão, } & \text { o } \\
\text { aumento } & \text { do } \\
\text { acesso } & \text { à } \\
\text { informação, } & \text { o } \\
\text { aumento da QV, a } & \text { ressignificação } \\
\text { relativa } & \text { à } \\
\text { ocupação } & \text { do } \\
\text { tempo livre } & \end{array}$ \\
\hline $\begin{array}{l}\text { Barrozo \& } \\
\text { Bizelli } \\
(2011) \\
\text { DB:18 }\end{array}$ & $\begin{array}{l}\text { Brasil/ } \\
\text { Arara } \\
\text { quara }\end{array}$ & 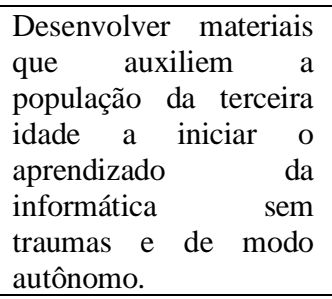 & 30 idosos & $\begin{array}{l}\text { Curso } r \text { de } \\
\text { Informática Básica } \\
\text { para a Terceira } \\
\text { Idade, } 60 \text { horas- } \\
\text { aula, distribuídas } \\
\text { em quatro horas } \\
\text { semanais, durante } \\
15 \text { semanas. }\end{array}$ & $\begin{array}{lr}\text { Análise } & \text { da } \\
\text { atividade } & \text { com } \\
\text { base em } & \text { livro } \\
\text { sobre TIC } & \end{array}$ & $\begin{array}{lr}\text { Foi } & \text { possível } \\
\text { concretizar } & \text { a } \\
\text { inclusão } & \text { digital } \\
\text { do idoso. } & \end{array}$ \\
\hline $\begin{array}{l}\text { Scoralik- } \\
\text { Lempke; } \\
\text { Barbosa } \\
\text { \&Mota } \\
\text { (2011) } \\
\text { DB: } 25\end{array}$ & $\begin{array}{l}\text { Brasil/ } \\
\text { Juiz } \\
\text { de } \\
\text { Fora }\end{array}$ & $\begin{array}{l}\text { Testar a hipótese de } \\
\text { que um processo de } \\
\text { alfabetização digital } \\
\text { melhora as capacidades } \\
\text { cognitivas de idosos, } \\
\text { mais especificamente } \\
\text { as memórias episódica } \\
\text { e de trabalho, a } \\
\text { metamemória e a } \\
\text { autoeficácia a em } \\
\text { memória. }\end{array}$ & $\begin{array}{l}38 \text { idosos } \\
\text { com } \\
\text { idades } \\
\text { médias de } \\
66,34 \\
\text { anos }\end{array}$ & $\begin{array}{l}\text { Três grupos, } 2 \\
\text { experimentais e } 1 \\
\text { controle. } \\
\text { grupos } \\
\text { experimentais } \\
\text { participaram de } 19 \\
\text { encontros } \\
\text { distribuídos ao } \\
\text { longo de cinco } \\
\text { meses. Eles foram } \\
\text { realizados uma vez } \\
\text { por semana, cada } \\
\text { um com duração } \\
\text { de duas horas. }\end{array}$ & $\begin{array}{l}\text { MEEM Lista de } \\
\text { palavras e } \\
\text { História } \\
\text { Subteste de } \\
\text { dígitos da escala } \\
\text { WAIS-III, MIA } \\
\text { (Metamemory } \\
\text { Adulthood } \\
\text { Questionnaire) e } \\
\text { MSEQ } \\
\text { (Memory Self } \\
\text { Efficacy } \\
\text { Questionnaire) }\end{array}$ & $\begin{array}{lr}\text { As alterações nos } \\
\text { processos } \\
\text { cognitivos dos } \\
\text { participantes não } \\
\text { puderam ser } \\
\text { atribuídas a esse } \\
\text { processo } \\
\text { educacional, uma } \\
\text { vez que foram } \\
\text { observadas } \\
\text { interações entre } \\
\text { tempo grupo em } \\
\text { somente três das } \\
\text { 26 variáveis } \\
\text { analisadas. }\end{array}$ \\
\hline $\begin{array}{c}\text { Cardoso et } \\
\text { al. (2014) } \\
\text { DB: } 23\end{array}$ & $\begin{array}{l}\text { Brasil/ } \\
\text { São } \\
\text { Luís }\end{array}$ & \begin{tabular}{l}
\multicolumn{3}{l}{ Analisar a percepção de } \\
idosos sob a \\
contribuição $\quad$ da \\
inclusão digital em sua \\
vida cotidiana e das
\end{tabular} & 14 idosos & $\begin{array}{l}\mathrm{O} \text { curso de } \\
\text { Informática Básica } \\
\text { foi oferecido junto } \\
\text { à Coordenadoria } \\
\text { do curso de }\end{array}$ & MEEM. & $\begin{array}{l}\text { Alto grau de } \\
\text { socialização e } \\
\text { diversos outros } \\
\text { benefícios. }\end{array}$ \\
\hline
\end{tabular}


Revista de Educação, Ciência e Tecnologia

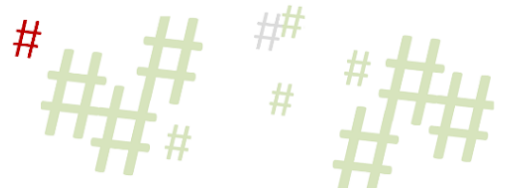

\begin{tabular}{|c|c|c|c|c|c|c|}
\hline & & $\begin{array}{l}\text { mudanças ocorridas em } \\
\text { suas concepções de } \\
\text { envelhecimento, após } \\
\text { frequentarem um curso } \\
\text { de inclusão digital. }\end{array}$ & & $\begin{array}{lr}\text { Sistema } & \text { de } \\
\text { Informação } & \text { e } \\
\text { aplicador por } \\
\text { alunos inseridos no } \\
\text { programa. } \\
\end{array}$ & & \\
\hline $\begin{array}{l}\text { Soares; } \\
\text { Santana \& } \\
\text { Rabelo } \\
(2015) \\
\text { DB: } 24\end{array}$ & $\begin{array}{c}\text { Brasil/ } \\
\text { Santo } \\
\text { Antôn } \\
\text { io de } \\
\text { Jesus }\end{array}$ & $\begin{array}{l}\text { Apresentar a } \\
\text { experiência de um } \\
\text { projeto de extensão de } \\
\text { oficinas de introdução à } \\
\text { informática associadas } \\
\text { ao treino cognitivo, } \\
\text { realizadas com idosos } \\
\text { da Universidade Aberta } \\
\text { à Terceira Idade na } \\
\text { cidade de Santo } \\
\text { Antônio de Jesus/BA. }\end{array}$ & 12 idosas & $\begin{array}{l}\text { Oficinas com } 20 \\
\text { encontros } \\
\text { semanais de } 2 \mathrm{hs} \text {. }\end{array}$ & $\begin{array}{l}\text { Exame } \\
\text { Cognitivo de } \\
\text { Addenbrooke. }\end{array}$ & $\begin{array}{l}\text { Maior inclusão } \\
\text { digital, } \\
\text { otimização } \\
\text { cognitiva e uma } \\
\text { maior descoberta } \\
\text { das suas próprias } \\
\text { potencialidades } \\
\text { em relação ao } \\
\text { aprendizado da } \\
\text { informática. }\end{array}$ \\
\hline $\begin{array}{l}\text { Tarallo \& } \\
\text { Sé } \\
(2016) \\
\text { DB: } 18\end{array}$ & Brasil & $\begin{array}{l}\text { Identificar as } \\
\text { motivações dos idosos } \\
\text { para aprender } \\
\text { informática e analisar } \\
\text { as estratégias e práticas } \\
\text { do uso do computador } \\
\text { em sala de aula. }\end{array}$ & $\begin{array}{c}\text { Idosos } \\
\text { acima de } \\
60 \text { anos. }\end{array}$ & $\begin{array}{l}\text { Curso } \\
\text { Informática. }\end{array}$ & $\begin{array}{l}\text { 1.Quest. Sobre } \\
\text { intimidade com } \\
\text { informática e } \\
\text { principais } \\
\text { dúvidas no uso } \\
\text { de tecnologias }\end{array}$ & 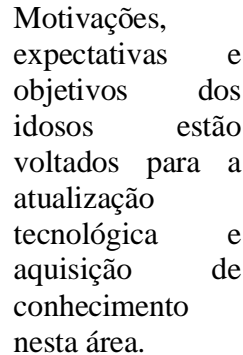 \\
\hline $\begin{array}{l}\text { Santos \& } \\
\text { Almêda } \\
\text { (2017) } \\
\text { DB:18 }\end{array}$ & $\begin{array}{l}\text { Brasil/ } \\
\text { Florân } \\
\quad \text { ia }\end{array}$ & $\begin{array}{lr}\text { Analisar o uso } & \text { das } \\
\text { tecnologias } & \\
\text { informação } & \text { de } \\
\text { comunicação } & \text { pelos } \\
\text { idosos do programa } \\
\text { PROEJA da cidade de } \\
\text { Florânia/RN no intuito } \\
\text { de compreender o } \\
\text { impacto do uso de tais } \\
\text { ferramentas na vida } \\
\text { desse público. }\end{array}$ & 12 idosos & $\begin{array}{l}\text { Curso } \\
\text { informática } \\
\text { PROEJA }\end{array}$ & $\begin{array}{l}\text { Entrevista in } \\
\text { loco com } \\
\text { questionários de } \\
\text { perguntas } \\
\text { abertas. }\end{array}$ & $\begin{array}{lr}\text { Os } & \text { fatores } \\
\text { motivacionais são } \\
\text { elementos } & \text { que } \\
\text { facilitam } & \text { o } \\
\text { processo } & \text { de } \\
\text { aprendizagem } & \text { do } \\
\text { uso r das } \\
\text { tecnologias r na } \\
\text { terceira idade. }\end{array}$ \\
\hline $\begin{array}{l}\text { Alvarenga; } \\
\text { Yassuda \& } \\
\text { Cachioni } \\
(2019) \\
\text { DB:20 }\end{array}$ & Brasil & $\begin{array}{l}\text { Descrever uma } \\
\text { intervenção de inclusão } \\
\text { digital, por meio da } \\
\text { utilização de tablets, } \\
\text { entre pessoas idosas. } \\
\text { Adicionalmente, } \\
\text { verificar o impacto } \\
\text { cognitivo. }\end{array}$ & 62 idosos & $\begin{array}{l}\text { Um programa de } \\
10 \text { semanas, } 15 \\
\text { horas semanais a } \\
\text { aprender um novo } \\
\text { conjunto de } \\
\text { competências } \\
\text { associadas ao } \\
\text { tablet. }\end{array}$ & $\begin{array}{l}\text { Entrevista } \\
\text { individual de } \\
\text { aproximadamen } \\
\text { te } 90 \text { minutos, e } \\
\text { ACE-r }\end{array}$ & $\begin{array}{l}\text { A participação no } \\
\text { grupo intervenção } \\
\text { resultou em } \\
\text { melhor } \\
\text { desempenho em } \\
\text { cognição geral, } \\
\text { atenção, funções } \\
\text { executivas, } \\
\text { habilidades } \\
\text { visuoespaciais e } \\
\text { diminuição de } \\
\text { sintomas } \\
\text { depressivo. }\end{array}$ \\
\hline
\end{tabular}

Fonte: elaborado pelos autores (2020)

A extração e síntese dos dados é uma tarefa executada posteriormente à fase de inclusão, especialmente para que sejam evidenciados os achados científicos e que estes sejam organizados de forma a atualizar o conhecimento da área de pesquisa sobre o envelhecimento humano. Outro aspecto importante é a da amostra coletada, que poderá trazer informações relevantes no comportamento das variáveis envolvidas nas pesquisas. 


\section{Resultados}

Após a busca nas bases de dados científicas, foram encontrados 436 estudos, que após exaustiva leitura e passando por diversos filtros baseados nos critérios de inclusão e exclusão, avaliação de qualidade baseada em ferramentas de checklist e leitura duplo cego com demais coautores, apenas nove foram eleitos para comporem esta revisão. Os nove estudos são brasileiros, em que participaram apenas pessoas idosas, que segundo o critério brasileiro, possuem idade maior ou igual a 60 anos, estudos que usaram diversos instrumentos em suas metodologias. Nos estudos incluídos nessa revisão é percebida a preocupação de investigar os efeitos de um programa de inclusão digital no desempenho cognitivo, na qualidade de vida e as motivações dos idosos.

Alguns estudos têm relacionado seus achados a uma redução dos níveis de ansiedade, ganho de confiança, autoeficácia e engajamento, a partir dos protocolos de intervenção com a inclusão digital (PEREIRA; NEVES, 2011; CARDOSO et al., 2014). Outros estudos (ORDONEZ; YASSUDA; CACHIONI, 2011; SOARES; SANTANA; RABELO, 2015; TARALLO; SÉ, 2016; SANTOS; ALMÊDA, 2017), concluíram que o curso de informática melhorou de forma significativa as funções executivas em geral, não concordando com os resultados da pesquisa de Scoralick-Lempke, Barbosa e Mota (2011) que, em seu estudo, não perceberam mudanças importantes ou encontraram melhorias pouco significativas nas funções da atenção, concentração e memória de trabalho.

Para cinco estudos, a melhoria na velocidade de processamento, na resolução de problemas e no raciocínio foi considerada significativa para os protocolos de intervenção de inclusão digital (ORDONEZ; YASSUDA; CACHIONI, 2011; PEREIRA; NEVES, 2011; SOARES; SANTANA; RABELO, 2015; TARALLO; SÉ, 2016; SANTOS; ALMÊDA, 2017). A melhoria na memória episódica foi constatada por três estudos (PEREIRA; NEVES, 2011; BARROZO; BIZELLI, 2011; CARDOSO et al., 2014), a melhoria na linguagem foi encontrada em dois estudos(ORDONEZ; YASSUDA; CACHIONI, 2011; SOARES; SANTANA; RABELO, 2015) que consideraram a melhoria como estatisticamente significativa e um estudo apresentou melhoria significativa para a função executiva (TARALLO; SÉ, 2016).

Em dois estudos foram encontradas melhorias pouco significativas para as habilidades visuoespaciais(ORDONEZ; YASSUDA; CACHIONI, 2011; SCORALICK-LEMPKE; BARBOSA; MOTA, 2011), mas outros três estudos encontraram uma melhoria para estas habilidades nos quais os idosos participam de programas de inclusão digital (SOARES; SANTANA; RABELO, 2015; TARALLO; SÉ, 2016; SANTOS; ALMÊDA, 2017). Considerando todos os aspectos cognitivos estudados, nenhum artigo apontou melhorias na função de orientação, a partir de intervenções baseadas em Tecnologia da Informação e Comunicação.

Além disto, tem sido relatadas alterações nos aspectos sociais e de autoconceito dos idosos, principalmente no que diz respeito ao aumento da consciência subjetiva do indivíduo, do reconhecimento de sua singularidade e de sua identidade social, bem como melhorias nas atividades de comunicação e seus efeitos nas redes sociais, trazendo benefícios nos relacionamentos interpessoais (BARROZO; BIZELLI, 2011; PEREIRA; NEVES, 2011; CARDOSO et al., 2014; SOARES; SANTANA; RABELO, 2015; TARALLO; SÉ, 2016. SANTOS; ALMÊDA, 2017). 


\section{Considerações Finais}

Os estudos na área têm demonstrado que as tecnologias de comunicação e de informação, em especial a Internet, estão possibilitando a inserção do idoso no mundo virtual e potencializando a interatividade e o acesso a informações, o qual vê ampliadas as oportunidades de se incluir novamente na sociedade. Dessa forma, pode ser verificado nos estudos incluídos nesta revisão, que a inclusão digital, a partir do uso das TIC, pode trazer benefícios em funções específicas importantes particularmente para os idosos, movimentando diversos aspectos relacionados a cognição e qualidade de vida, considerando que o envelhecimento é biológica e psicologicamente diferente para cada indivíduo.

$\mathrm{Na}$ análise geral, entretanto, apenas um estudo confirmou um ganho efetivo para a maioria das funções cognitivas dos idosos e outros quatro estudos concordam com a hipótese de que os programas de inclusão digital, que se utilizam das várias formas de tecnologia, melhoram a cognição dos idosos. Além destes resultados, não foram encontrados estudos sobre a permanência destes efeitos benéficos a longo prazo, como também não foram encontrados resultados quanto ao possível tempo de retardamento do declínio cognitivo, o que poderia ser possível a partir dos protocolos de intervenção em idosos saudáveis.

A produção científica publicada sobre o assunto até este momento, considerando os critérios de inclusão e exclusão deste estudo, não pode ser considerada muito ampla e nem mesmo conclusiva quanto aos efeitos das Tecnologia da Informação e Comunicação na cognição da população idosa, sendo necessário que sejam desenvolvidos novos estudos, sendo estes com maior amplitude longitudinal, proporcionando um maior tempo de análise e percepção de possíveis efeitos protetivos dos métodos de inclusão digital para esta população.

\section{Financiamento e agradecimentos}

O presente trabalho foi realizado com apoio da Coordenação de Aperfeiçoamento de Pessoal de Nível Superior - Brasil (CAPES) - Código de Financiamento 001.

\section{Referências}

ALVARENGA, G. M., YASSUDA, M.; CACHIONI, M. Inclusão digital com tablets entre idosos: metodologia e impacto cognitivo. Psicologia, Saúde \& Doenças, v. 20, n.2, p. 384401, 2019. DOI: http://dx.doi.org/10.15309/19psd200209

ARGIMON, I. I. L.; STEIN, L. M. Habilidades cognitivas em indivíduos muito idosos: um estudo longitudinal. Cadernos de Saúde Pública, v.21, n.1, p. 64-72, 2005. ISSN 1678-4464.

BARROZO, S.; BIZELLI, M. H. S. S. A informática ao alcance do idoso: desenvolvimento de material específico. Repositório Unesp, Campus Araraquara, p. 6259- 6266, 2011. ISSN: 2236-97082.

CARDOSO, R. et al. Os benefícios da informática na vida do idoso. Computer on the Beach, p. 340- 349, 2014.DOI: https://doi.org/10.14210/cotb.v0n0.pp.340-349

DOWNS, S. H.; BLACK, N. The feasibility of creating a checklist for the assessment of the methodological quality both of randomized and non-randomised studies of health care 
interventions. Journal of Epidemiology and Community Health, v. 52, n.6, p. 377-384, 1998. ISSN PMCID: PMC1756728.

FONSECA, V. da. Papel das funções cognitivas, conativas e executivas na aprendizagem: uma abordagem neuropsicopedagógica. Revista Psicopedagogia, v.31, n. 96, p.236- 253, 2014. ISSN 0103-8486.

FUJITA, L. S. Comparação dos domínios da cognição, autoeficácia e domínios da qualidade de vida entre idosas participantes, não participantes e concluintes do Curso de Informática. Curitiba: Universidade Federal do Paraná. Acesso em 16 de Abr de 2021.

FLORES-GOMES, G.; GOMES, F. R. H.; GASPAROTTO, G.S.; OLIVEIRA, V.;

VAGETTI, G. C. Quality of life of elderly: effects of a digital inclusion protocol in South Brazil. Research, Society and Development, v. 9, n.7, p.1-15, 2020. DOI:

http://dx.doi.org/10.33448/rsd-v9i7.2756

GALVÃO, T.F.; et al.Principais itens para relatar Revisões sistemáticas e Meta-análises: A recomendação PRISMA. Epidemiologia e Serviços de Saúde, v.24, n.2, p.335-342, 2015. https://doi.org/10.5123/S1679-49742015000200017

KACHAR, V. Terceira idade e informática: aprender revelando potencialidades. São Paulo: Cortez, 2003. 206p.

KACHAR, V. Envelhecimento e perspectivas de inclusão digital. Revista Kairós Gerontologia, São Paulo, v.13, n.2, p. 131-147, nov. 2010. DOI: https://doi.org/10.23925/2176-901X.2010v13i2p\%25p

KRAMPE, T. T. Lifes panchanges in multi- tasking: concurrent walking and memory search in children, young and older adults. Gait Posture, v.33, n.3, p.401-4015, 2011. DOI: 10.1016 / j.gaitpost.2010.12.012.

LEMOS, C. E. S.; SANTOS, I. C. L.; SILVA, N. A.; PINTO, T. H. C. Laboratório de Multimídia da Universidade para a Terceira Idade: a inclusão digital como estímulo às habilidades cognitivas e sociabilidade dos idosos. In: X Congresso Nacional de Educação Curitiba: I Seminário Internacional de Representações Sociais, Subjetividade e Educação. Anais... Curitiba, 2011. p. 12405-12412.

MALTA, M. et al. Iniciativa STROBE: subsídios para a comunicação de estudos observacionais. Revista Saúde Pública, v,44, n.3, p. 559-65, 2010.https://doi.org/10.1590/S0034-89102010000300021

NUNES, M. V. R. D. S. Envelhecimento Cognitivo: principais mecanismos explicativos e suas limitações. Cadernos de saúde, Lisboa, Portugal, v. 2, n. 2, p. 19-29, 2009. ISSN 1647 0559. Disponível em: <http://hdl.handle.net/10400.14/9880>. Acesso em: 20 jul. 2019.

OLIVEIRA, R. de C. da S; SCORTEGAGNA, P. A.; OLIVEIRA, F. da S. Universidades Abertas a terceira idade: delineando um novo espaço educacional para o idoso. Revista HISTEDBR On-line, Campinas, n. 64, p. 343- 358, set. 2015. DOI: https://doi.org/10.20396/rho.v15i64.8641945 
ORDONEZ, T. N.; YASSUDA, M. S.; CACHIONI, M. Elderly online: Effects of a digital inclusion program in cognitive performance. Archives of Gerontology and Geriatrics, São Paulo, n. 53, p. 216-219, 2011.DOI: 10.1016/ j.archger.2010.11.007

PARENTE, M. M. P.; OLIVEIRA, A. A.; JAEGER, A. Cognição e envelhecimento. São Paulo: Artmed, 2006.

PEREIRA, C; NEVES, R. Os idosos e as TIC - competências de comunicação e qualidade de vida. Revista Kairós Gerontologia, v.14, n.1, p.05-26, março 2011. ISSN 2176-901X

SANTOS, R. F.dos; ALMÊDA, K .A. O envelhecimento humano e a inclusão digital: análise do uso das Ferramentas Tecnológicas pelos idosos. Ciência da Informação, v. 4, n. 2, p. 5968, maio/ago. 2017. ISSN 2358-0763

SAMPAIO, R. F.; MANCINI, M. C. Estudos de revisão sistemática: um guia para síntese criteriosa da evidência científica. Rev. bras. Fisioter., v.11, n.1, p.83-89,

2007.https://doi.org/10.1590/S1413-35552007000100013

SCORALICK-LEMPKE, N. N.; BARBOSA, A. J. G.; MOTA, M. M. P. E. D. Effects of Digital Literacy Process in Elderly Cognition. Psicologia: Reflexão e Crítica, Juiz de Fora, v. 4, n. 25, p. 774-782, 2011.ISSN 0102-7972.

SILVEIRA, M. M. DA. Desempenho cognitivo, estado emocional, qualidade de vida e habilidade motora manual de idosos participantes de oficinas de informática. 2015. 158f. Tese (Doutorado), Pós-Graduação em Gerontologia Biomédica, Instituto de Geriatria e Gerontologia. Porto Alegre, PUCRS, 2015.

SOARES, Z. F; SANTANA, E. F de; RABELO, D. F. Iniciação à informática associada ao treino cognitivo com idosos. Revista Ciência e Extensão. v.11, n.3, p. 155-167, 2015. ISSN:1679-4605.

TARALLO, R. dos S.; SÉ, E. V. G. Letramento digital no ensino de informática para idosos. Revista Intellectus, n. 36, v. 4, p. 53-66, 2016.http://dx.doi.org/10.21116/2016.6.

WILD, K. V.;et al. Computer-related self-efficacy and anxiety in older adults with and without mild cognitive impairment. Alzheimer's \& Dementia, v. 8, p. 544-552, 2012.DOI: 10.1016 / j.jalz.2011.12.008.

VAGETTI, G. C.; et al. Domínios da qualidade de vida associados à percepção de saúde: um estudo com idosas de um programa de atividade física em bairros de baixa renda de Curitiba, Paraná, Brasil. Ciênc. Saúde Coletiva, n.18, v.12, p. 3483-3493, 2013. DOI:10.1590/S141381232013001200005.

Recebido em dezembro de 2020.

Aprovado em maio de 2021. 